\title{
A REMARK ON INCOMPARABLE ULTRAFILTERS IN THE RUDIN-KEISLER ORDER
}

\author{
EVA BUTKOVIČOVÁ
}

(Communicated by Dennis Burke)

\begin{abstract}
If $2^{<c}>\mathbf{c}$ and $p$ is an ultrafilter on $\omega$ of character $\mathbf{c}$ then there exist many ultrafilters that are incomparable with $p$ in the Rudin-Keisler order.
\end{abstract}

The Rudin-Keisler order $\leq_{\mathrm{RK}}$ on $\omega^{*}$ is defined as $p \leq_{\mathrm{RK}} q$ iff there exists a function $f: \omega \rightarrow \omega$ such that $\beta f(q)=p$, hence $A \in p \equiv f^{-1}[A] \in q$. Problem 19 from [vM] and problem 48 from [HvM] asks: does there exist for every $p \in \omega^{*}$ a $q \in \omega^{*}$ such that $p$ and $q$ are incomparable in the RudinKeisler order? A partial answer is given by Hindman in [H]: if $p$ has character c and either $\mathbf{c}$ is singular or all predecessors of $p$ are of character $\mathbf{c}$ then such a $q$ exists.

We shall obtain a stronger result as a corollary to the following theorem.

Theorem. If $\kappa<\mathbf{c}$ and $p \in \omega^{*}$ has character $\mathbf{c}$ then there exist $2^{\kappa}$ ultrafilters which are not $\leq_{\mathbf{R K}^{-}}$-greater than $p$.

Before we give the proof we fix some notation.

Let $F$ denote the Fréchet (cofinite) filter on $\omega$.

For $\mathscr{F} \subseteq \mathscr{P}(\omega)$ put

$$
\langle\not \mathscr{F}\rangle=\left\{A \subseteq \omega: \exists H \in[\mathscr{F}]^{<\omega} \bigcap H \subseteq A\right\} .
$$

We call the system $\mathscr{F}$ centered if $|\cap H|=\omega$ for every finite $H \subseteq \mathscr{F}$.

Clearly, if $\mathscr{F}$ is centered then $\langle\langle\mathscr{F}\rangle\rangle$ is the filter generated by $\mathscr{F}$.

Let ${ }^{\kappa} 2$ denote the product of $\kappa$ copies of 2 , or equivalently the set of functions from $\kappa$ to 2 . Of course $\left|{ }^{\kappa} 2\right|=2^{\kappa}$.

Proof. We find $2^{\kappa}$ distinct ultrafilters that are not $\leq_{\mathbf{R K}}$-greater than $p$.

Let $\left\{f_{\gamma}: \gamma<\mathbf{c}\right\}$ be an enumeration of the set of functions from $\omega$ to $\omega$. We shall construct by induction on $\gamma<\mathbf{c}$ filters $\mathscr{F}_{g}^{\gamma}$ for $g \in{ }^{\kappa} 2$ such that

(1) $\chi\left(\mathscr{F}_{g}^{\gamma}\right) \leq \kappa+\gamma$,

Received by the editors February 6, 1990.

1980 Mathematics Subject Classification (1985 Revision). Primary 04A20, 03E35.

Key words and phrases. Ultrafilters, Rudin-Keisler order. 
(2) there is a set $G \in \mathscr{F}_{g}^{\gamma+1}$ such that $f_{\gamma}[G] \notin p$, and

(3) if $\gamma<\delta<\mathrm{c}$ then $\mathscr{F}_{g}^{\gamma} \subseteq \mathscr{F}_{g}^{\delta}$.

We shall see that (1) ensures (2) can be satisfied. Note that (2) implies any ultrafilter $q_{g}$ extending $\bigcup_{\gamma<\mathrm{c}} \mathscr{F}_{g}^{\gamma}$ will not be $\leq_{\mathbf{R K}}$-above $p$.

Let $\left\{A_{\beta}^{i}: \beta<\kappa, i \in 2\right\}$ be an independent family of sets. This means that for every $\beta<\kappa$ we have $A_{\beta}^{0} \cap A_{\beta}^{1}=\varnothing$ and $A_{\beta}^{0} \cup A_{\beta}^{1}=\omega$ and that for every finite partial function $\varphi: \kappa \rightarrow 2$ the intersection $\bigcap_{\beta \in \operatorname{dom} \varphi} A_{\beta}^{\varphi(\beta)}$ is infinite. Such families exist; see e.g., [vM, Lemma 3.3.2].

For every $g \in{ }^{\kappa} 2$ we consider the family $\mathscr{F}_{g}=\left\{A_{\beta}^{g(\beta)}: \beta<\kappa\right\}$. Each family $\mathscr{F}_{g}$ is centered since we only took sets from the independent family. We start with the distinct filters

$$
\mathscr{F}_{g}^{0}=\left\langle\left\langle F \cup \mathscr{F}_{g}\right\rangle\right\rangle \quad \text { for } g \in{ }^{\kappa} 2 .
$$

Now suppose we are at stage $\gamma<\mathrm{c}$ and take $g \in{ }^{\kappa} 2$. Here we use part of Hindman's construction. Since $\chi(p)=\mathbf{c}$ the system $\left\{f_{\gamma}[B]: B \in \mathscr{F}_{g}^{\gamma}\right\}$ does not generate $p$. Hence there is a set $E$ such that $\omega-E \in p$ and $\left\{f_{\gamma}[B]: B \in\right.$ $\left.\mathscr{F}_{g}^{\gamma}\right\} \cup\{E\}$ is centered. It follows that $\mathscr{F}_{g}^{\gamma} \cup\left\{f_{\gamma}^{-1}[E]\right\}$ is also centered and we can add $G=f_{\gamma}^{-1}[E]$ to $\mathscr{F}_{g}^{\gamma}$, i.e., we let $F_{g}^{\gamma+1}=\left\langle\left\langle\mathscr{F}_{g}^{\gamma} \cup\left\{f_{\gamma}^{-1}[E]\right\}\right\rangle\right.$.

In case $\gamma$ is a limit we let $\mathscr{F}_{g}^{\gamma}=\bigcup_{\delta<\gamma} \mathscr{F}_{g}^{\delta}$.

If at any moment $\mathscr{F}_{g}^{\gamma}$ is an ultrafilter then we can stop. It cannot possibly be $\leq_{\mathbf{R K}}$-above $p$ since its character is smaller than that of $p$.

Since clearly every ultrafilter has at most $\mathbf{c} \leq_{\mathbf{R K}}$-predecessors, the following is an immediate consequence of our theorem.

Corollary. If $\kappa<\mathbf{c}$ is such that $2^{\kappa}>\mathbf{c}$ then for every $p \in \omega^{*}$ of character $\mathbf{c}$ there exist $2^{\kappa}$ ultrafilters that are $\leq_{\mathbf{R K}}$-incomparable with $p$.

Remarks. 1 . Since $2^{c f} \mathbf{c}>\mathbf{c}$ our corollary also covers the case when $\mathrm{c}$ is singular.

2. Another easy consequence of our corollary is that if one assumes that $2^{\kappa}>\mathrm{c}$ for some $\kappa<\mathrm{c}$ and that there is a $p \in \omega^{*}$ such that $\chi(r)=\mathrm{c}$ for all $r \leq_{\mathbf{R K}} p$ then for every $u \in \omega^{*}$ there is a $v \in \omega^{*}$ that is $\leq_{\mathbf{R K}}$-incomparable with it. (Given $u$, if the character of $u$ is $\mathbf{c}$, the corollary applies. Otherwise $p$ is not $\leq_{\mathbf{R K}}$-comparable to $u$.)

\section{REFERENCES}

[HvM] K. P. Hart and J. van Mill, Open problems on $\beta \omega$, Problems in Topology (to appear).

$[\mathrm{H}] \quad \mathrm{N}$. Hindman, Is there a point of $\omega^{*}$ that sees all others?, Proc. Amer. Math. Soc. 104 (1988), 1235-1238.

[vM] J. van Mill, An introduction to $\beta \omega$, Handbook of Set-Theoretic Topology (K. Kunen and J. E. Vaughan, eds.), North-Holland, Amsterdam, 1984, pp. 504-568.

Mathematical Institute, Slovak Academy of Sciences, Jesenná 5, 04154 Košice, Czechoslovakia 